Accretion Phenomena and Related Outflows, IAU Colloquium 163

ASP Conference Series, Vol. 121, 1997

D.T. Wickramasinghe, L. Ferrario, and G.V. Bicknell, eds.

\title{
ASCA observation of 4U1624-49
}

\author{
Lorella Angelini ${ }^{1}$
}

LHEA, Code 660, NASA/GSFC, Greenbelt, MD 20771

\author{
Arvind Parmar \\ ESTEC/SSD/ESA, 2200 AG Noordwijk, The Netherlards \\ Nicholas White \\ LHEA, Code 660, NASA/GSFC, Greenbelt, MD 20771
}

\begin{abstract}
ASCA observations of the LMXRB 4U1624-49 reveal the presence of extended emission surrounding the X-ray source probably due to a dust scattered halo. The halo spectrum can account for the low energy excess seen in the dip spectrum.
\end{abstract}

\section{Observation and Results}

Several low mass X-ray binaries exhibit periodic irregular dips in their X-ray lightcurves (see White, Nagase, \& Parmar 1995 for a review). 4U1624-49 shows perhaps the most dramatic dipping behaviour, with a dip recurrence period of $21 \mathrm{hr}$ and dip activity lasting $\sim 6 \mathrm{hr}$. The X-ray dipping phenomenon is understood as obscuration of a central X-ray source due to material located at the outer edge of the disk where the gas stream from the companion hits the accretion disk. The simple cold-absorption model fails to describe the dip spectrum, which instead shows an excess of counts at low energy when compared to the continuum spectrum. ASCA observed 4U1624-49 for one day in March 1993. Dips detected at the beginning and the end of the observation are not total. Erratic variability accompanied by partial hardening is seen in the first part of the dip, while substantial softening is seen in the lower intensity level of the dip. Either a multicolor disk blackbody (MDB) or a cutoff power law (CPL) model describes the out of the dip spectrum (OD) although the fit is formally unacceptable $\left(\chi^{2} /\right.$ d.o.f. of 1.45 , spectrum extracted in 3 arcmin radius $)$. The measured $\mathrm{N}_{H}$ ranges between $6-8 \times 10^{22}$ atoms $\mathrm{cm}^{-2}$. Using either MDB or CPL model to fit the low intensity dip spectrum (DP) gives a soft excess bewteen 1-3 keV. The dip spectrum also shows line emission which is consistent either with a $6.4 \mathrm{keV}$ broad line or with two narrow lines at 6.2 and $6.9 \mathrm{keV}$.

The soft excess is seen in most of the dip sources and different explanations have been proposed : ionization of the absorbing material by the central X-ray source; scattering emission in an accretion disk corona (ADC); rapid variations in absorption on a timescale faster than the accumulation time for the spectra; reduced abundances in the absorbing material. The dip spectrum has been successfully modeled by two components a direct (absorbed) and scattered into the

\footnotetext{
${ }^{1}$ Universities Space Research Association
} 
line of sight (unabsorbed) where the relative flux between the two components gives an indication of the absorbing material. An alternative two component model, one extended and one compact (used for 4U1755-338 and 4U1624-46)
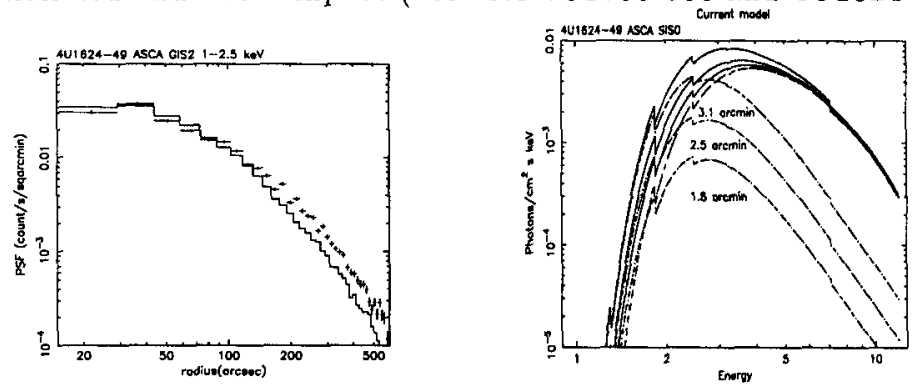

Figure 1. Left: GIS2 surface brightness compared to the prediction for a point source. Right: The individual contribution of the two component model spectra for the 3 extracted radii. The dotted line is the halo component, the dash-dot line the MDB component and the solid line the sum of the two components

explains the dip spectrum by obscuration of the compact component (Church et al. 1995). Another important effect is scattering off interstellar dust grains, which can cause a halo up to several arc minutes around the X-ray source, depending on the overall absorption. The spectrum of such a component is expected to be softer than the direct emission. Since 4U1624-67 shows an absorbed spectrum, the low energy excess in the dip could be explained by the presence of a dust scattered halo. The source surface brightnesses (crosses, Fig 1) in the GIS image (1-2.5 keV) deviates noticeably at radii greater than 120 arcsec when compared to the distribution expected for a point source (solid line). The contribution of this extra extended component in the energy band $1-2.5 \mathrm{keV}$ is about $20 \%$ at $9.5 \operatorname{arcmin}, 6 \%$ at 3.5 arcmin and $1 \%$ at 2 arcmin. Since the halo should not vary, the amount of the soft excess should be constant in the OD and DP spectrum when extracted from the same region. By contrast spectra extracted with increasing region sizes should show different levels of contamination due to the halo. To model the halo component an additional power law was added to model. The OD and DP spectra were simultaneously and successfully fit with the slope and normalization of the power law component coupled across the spectra. The halo contribution to the total flux is $4 \%$ and $18 \%$ for $\mathrm{OD}$ and DP spectra respectively. A second set of spectra taken during the dip were extracted using region size of $3,2.5$ and 1.8 arcmin radius. The 3 spectra were simultaneously fit to a two component model: a MDB to account for the 4U1624-49 emission and a power law for the halo. The contribution of the halo component is $10 \%$ at $2.5 \mathrm{arcmin}$ and $<3 \%$ at $1.8 \mathrm{arcmin}$. These results suggest that the soft excess can be explained by a scattered component due to an interstellar dust halo instead of an accretion disk corona.

\section{References}

Church et al., 1995, A\&A.

White,N.E.,Nagase, F.,\& Parmar, A.N. 1995, in Accretion Driven X-ray Binaries, eds. W. Lewin \& J. van Paradijs. Cambridge University Press. 